in non-ST-segment Elevation myocardial infarction-2 (DISPERSE-2) is a randomized, double-blind trial designed to investigate the safety, tolerability and efficacy of two different doses of AZD6140 given with aspirin and to compare these treatment regimens with clopidogrel plus aspirin in patients with NSTE-ACS. The safety analysis cohort included 984 participants, with 491 (50\%) individuals receiving AZD6140 for 12 weeks, $243(25 \%)$ receiving it for 8 weeks, and $250(25 \%)$ receiving it for 4 weeks.

Cannon et al. concentrated on the safety profile of AZD6140, using the Kaplan-Meier scale to assess the rate of major or minor bleeding events at 4 weeks after the initiation of treatment. Major or minor bleeding events were detected in $8.1 \%$ of the clopidogrel group, $9.8 \%$ of the group receiving $90 \mathrm{mg}$ AZD6140, and $8.0 \%$ of the group receiving $180 \mathrm{mg}$ AZD6140. Within these groups, the major bleeding rates were $6.9 \%, 7.1 \%$ and $5.1 \%$, respectively. Although there was a trend towards fewer myocardial infarctions in the AZD6140 treatment groups, particularly in the higher-dose group, this trend did not reach statistical significance.

In a companion study, nested within the main DISPERSE-2 trial, Storey et al. compared the antiplatelet activity of AZD6140 with that of clopidogrel in 91 patients, 44 of whom had previously taken clopidogrel and 45 of whom had never taken this drug. Monthly measurements of platelet aggregation revealed that AZD6140 was a significantly more potent inhibitor of aggregation (at both doses) than was clopidogrel and that the effects of AZD6140 were dose related. Even patients previously treated with clopidogrel had superior inhibition of platelet aggregation with AZD6140 than with clopidogrel.

Data from the overall DISPERSE-2 study show that, in this initial experience, there was no significant difference between AZD6140 and clopidogrel in terms of the rate of major bleeding events, and the authors conclude that AZD6140 shows similar safety and tolerability to clopidogrel. Storey et al. note that AZD6140 seems to be a more potent inhibitor of platelet aggregation than clopidogrel in patients with NSTE-ACS, providing greater levels of inhibition even in patients already responding to clopidrogrel. These results suggest that the potential benefits of AZD6140 should be explored in larger trials involving patients with ACS with and without ST-segment elevation.
Original articles Cannon CP et al. (2007) Safety, tolerability, and initial efficacy of AZD6140, the first reversible oral adenosine diphosphate receptor antagonist, compared with clopidogrel, in patients with non-ST-segment elevation acute coronary syndrome: primary results of the DISPERSE- 2 trial. JAm Coll Cardiol 50: 1844-1851

Storey RF et al. (2007) Inhibition of platelet aggregation by AZD6140, a reversible oral $\mathrm{P}_{2} \mathrm{Y}_{12}$ receptor antagonist, compared with clopidogrel in patients with acute coronary syndromes. J Am Coll Cardiol 50: 1852-1856

\section{Could changes in intestinal function and morphology underlie chronic heart failure?}

Chronic heart failure (CHF) is a state of chronic inflammation, but the origins of the inflammatory state are unclear. One parameter that might be involved in the generation of this state is lipopolysaccharide, also called endotoxin. This cell-wall component of Gram-negative bacteria can cross the gut wall and enter the general circulation if normal barrier function is impaired. It has been suggested that an enhanced intestinal bacterial biofilm in patients with $\mathrm{CHF}$ might additionally contribute to elevated plasma concentrations of lipopolysaccharide. This rise in lipopolysaccharide levels might then activate monocytes and macrophages to release proinflammatory mediators and, thus, stimulate inflammation.

To test this theory, Sandek et al. evaluated 22 patients with $\mathrm{CHF}$ and compared the function and morphology of their guts with those of 22 controls. Patients with CHF had significantly thicker bowel walls in the terminal ileum, the ascending, transverse and descending colon, and in the sigmoid, than did controls. In addition, patients with CHF showed increased intestinal permeability and decreased D-xylose absorption, which strongly suggested bowel ischemia. Furthermore, the mucus of patients with CHF contained larger numbers of adherent bacteria than did that of control individuals, indicating the presence of an augmented biofilm.

The authors recognize that further investigation is warranted, but suggest that increased permeability of the intestine in patients with $\mathrm{CHF}$, and the presence of an augmented bacterial biofilm, could contribute to the chronic inflammatory state and the malnutrition long observed in patients with $\mathrm{CHF}$.

Original article Sandek A et al. (2007) Altered intestinal function in patients with chronic heart failure. J Am Coll Cardiol 50: 1561-1569 